\title{
Properties of Reaction Intermediates from Unzipping Nanotubes via the Diketone Formation: A Computational Study
}

\author{
Takashi Yumura and Toshiyuki Kanemitsu \\ Department of Chemistry and Materials Technology, Kyoto Institute of Technology, Matsugasaki, Sakyo-ku, Kyoto 606-8585, Japan \\ Correspondence should be addressed to Takashi Yumura, yumura@chem.kit.ac.jp
}

Received 13 October 2011; Accepted 18 March 2012

Academic Editor: Sulin Zhang

Copyright (c) 2012 T. Yumura and T. Kanemitsu. This is an open access article distributed under the Creative Commons Attribution License, which permits unrestricted use, distribution, and reproduction in any medium, provided the original work is properly cited.

\begin{abstract}
We investigated properties of an armchair nanotube attached by specific numbers of diketone groups along the tube axis using density functional theory (DFT) calculations. The results from DFT calculations show that multiple diketone attachments into an armchair nanotube cleave the CC bonds along the tube axis, forming a large hole. Then, the six-membered rings surrounding the hole are planarized, and zigzag edges appear. Due to these geometrical changes, the functionalized armchair nanotubes exhibit properties similar to those in corresponding graphene ribbons with zigzag edges. For example, diketone-attached nanotubes have a spin-polarized ground state with frontier orbitals whose amplitudes are localized at diketone $\mathrm{O}$ atoms. As a consequence of the existence of the localized orbitals, unpaired electrons appear only on the diketone $\mathrm{O}$ atoms in an armchair nanotube.
\end{abstract}

\section{Introduction}

Honeycomb carbon sheets $[1,2]$ are contained in both single-walled carbon nanotubes $[3,4]$ and single-layer graphite, also called graphene [5]. The two carbon allotropes, which use the same building blocks, exhibit similar and unique electronic properties resulting from small band gaps $[1,2]$. Although the unique properties primarily come from $\pi$ orbitals in the frontier orbital region, there are some differences in their electronic properties. The differences originate from the dimensionality of the nanocarbon materials. In fact, one-dimensional nanotubes exhibit metallic or semi-conducting character, depending on how the honeycomb sheet rolls up [6-9]. The properties of thin strips of graphene (graphene-ribbons) are strongly influenced by their width and edge structures (armchair- and zigzag-edges) [10-23]. In particular, zigzagnanoribbons have radical character, which is localized at their edges.

To demonstrate the ability to connect nanotubes and graphene [24-30], Kosynkin et al. successfully converted a nanotube into a nanoribbon via a chemical treatment [26]. In this intriguing experiment [26], $\mathrm{KMnO}_{4}$ oxidizes a nanotube and unzips the nanotube longitudinally to form a nanoribbon, as shown in Figure 1. In the proposed reaction mechanism, $\mathrm{KMnO}_{4}$ binds initially to a CC bond in the nanotube. After the binding, one diketone group is formed by cleaving the attached CC bond. Because the first diketone formation distorts the neighboring CC bonds, the distorted CC bonds become a suitable site for the second $\mathrm{KMnO}_{4}$ addition. After a sequence of oxidation reactions, the $\mathrm{CC}$ bonds of the tube are successfully cleaved along the tube axis. Although their proposal appears plausible and is consistent with the experimental findings, atomic-scale views of the reaction intermediates, which include a nanotube attached by multiple diketone groups, are still required.

For this purpose, computer simulations based on density functional theory (DFT) [31-38] can provide insights into the reaction mechanism. Although several computational studies have focused on unzipping nanotubes with and without $\mathrm{H}$-atom termination [31-35], there are a few reports discussing those created by the addition of oxygen pairs $[36,37]$. For example, Rangel et al. used DFT calculations to investigate the sequential addition of oxygen pairs to a model with a finite-length $(5,5)$ tube. The model, which included 100 atoms, has a tube length of $\sim 10 \AA$. Based on the tube length, the maximum number of oxygen pairs attached to 

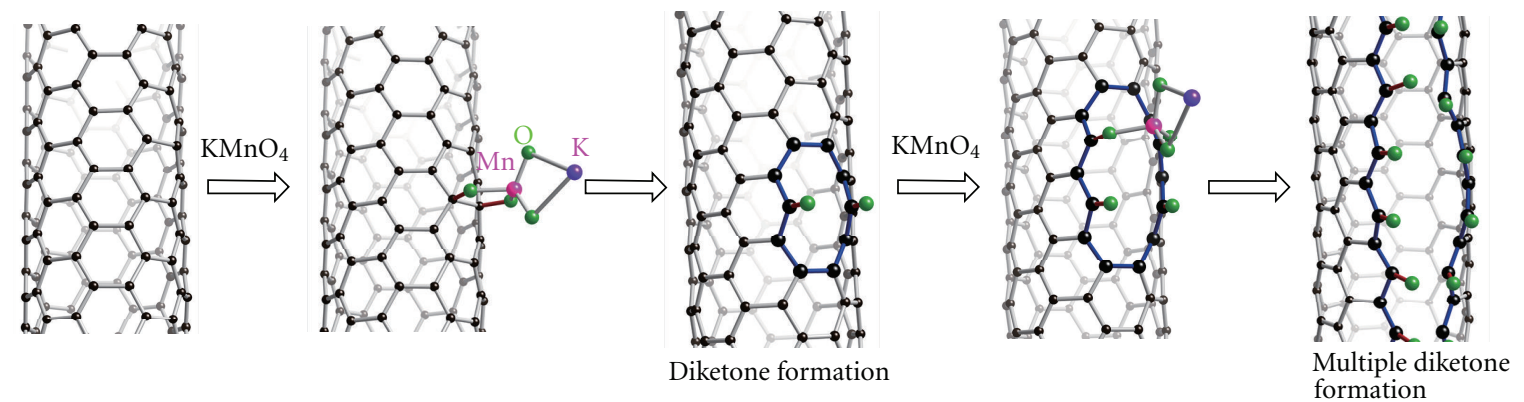

FIGURE 1: Reaction mechanism for unzipping a nanotube by $\mathrm{KMnO}_{4}$, proposed in [26]. The optimized structures for a nanotube $((5,5)$ nanotube) attached by $\mathrm{KMnO}_{4}$, and a one-diketone-attached nanotube bound by second $\mathrm{KMnO}_{4}$ were obtained in the PW91 calculation.

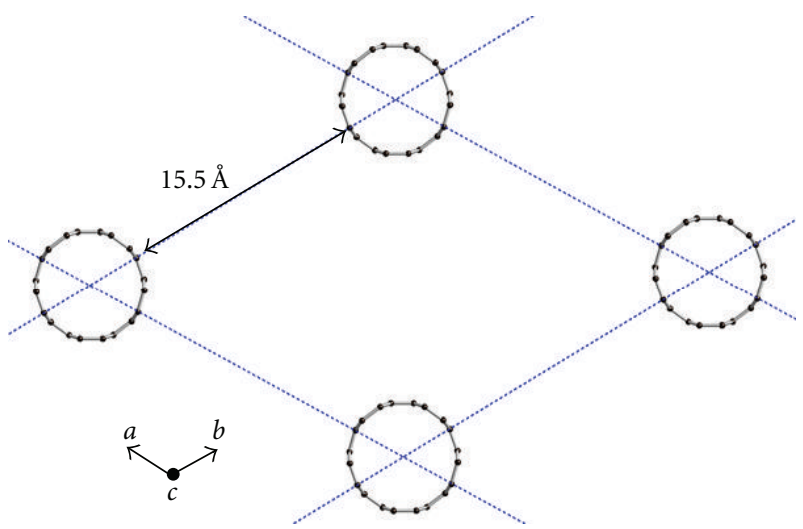

(a)

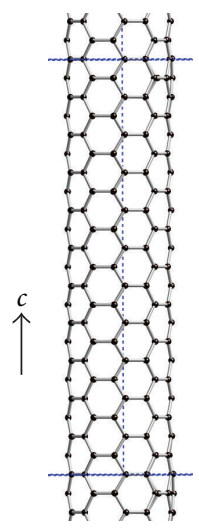

(b)

Figure 2: (a) Cross-section of the hexagonal unit cell for the $(5,5)$ nanotube in periodic boundary condition (PBC) calculations, given by blue hashed lines. (b) Side-view of the nanotube along the tube axis (the $c$ vector). Its supercell was given by blue-hashed lines.

the tube $\left(N_{\mathrm{op}}\right)$ can be as many as 5 . According to the DFT calculations, cleaving the tube CC bonds by diketone formation was found for $N_{\text {op }}$ larger than 3. Although their DFT findings can partially explain the experimental results, the structural features of the oxygen-pair attached nanotubes are artificially affected by the model size. In fact, the separation between the carbon atoms attached by oxygen pairs is influenced by the tube edges. Furthermore, attached oxygen atoms cannot line up along the tube axis at regular intervals. Because these structural features have a strong impact on the electronic properties, detailed insight into the properties of diketone-attached nanotubes is still required.

To improve our understanding of the conversion of nanotubes into nanoribbons by multiple diketone attachments, we employed DFT calculations for a sufficiently long tube model and an infinite-length model using a periodic boundary condition (PBC). In the current study, we focused on how the properties of diketone-attached nanotubes change as a function of the degree of oxidation (i.e., the number of diketones formed on a nanotube surface $\left.\left(N_{\mathrm{op}}\right)\right)$. It is expected that the diketone-attached nanotubes have properties in between a pristine nanotube and the resultant graphenenanoribbon. Thus, we focused on how a nanotube becomes planar via a sequence of diketone formation and how the planarization affects the electronic properties.

\section{Method of Calculation}

The DFT calculations used in the current study are based on the Perdew-Wang (PW91) functional [39]. When some numbers of diketones are formed on a CC bond perpendicular to the axis of a $(5,5)$ armchair nanotube, the CC bonds at the binding sites are proposed to be cleaved [26]. After cleaving the attached CC bonds along the axis, zigzag edges appeared at the binding sites. Previous theoretical studies indicate that a spin-polarized solution for the zigzag nanoribbons with attached ketone groups is energetically stable relative to its spin-unpolarized solution [18-20]. We performed both spin-polarized and spin-unpolarized calculations to obtain local minima of the diketone-attached nanotubes.

In the PBC calculations implemented in the Vienna ab initio Simulation Package (VASP v.4.6) [40], we employed a hexagonal supercell in Figure 2. The supercell contains 200 $\mathrm{C}$ atoms in the $(5,5)$ nanotube and some number of oxygen pairs. In the current study the number of attached oxygen pairs $\left(N_{\text {op }}\right)$ ranges from 1 to 7 . The kinetic energy cutoff of the plane-wave basis set is $349.5 \mathrm{eV}$ with ultrasoft Vanderbilt-type pseudopotentials [41]. We allowed full geometry relaxation in the axial direction, but separations between adjacent tubes are fixed at $15.5 \AA$ (Figure 2) 


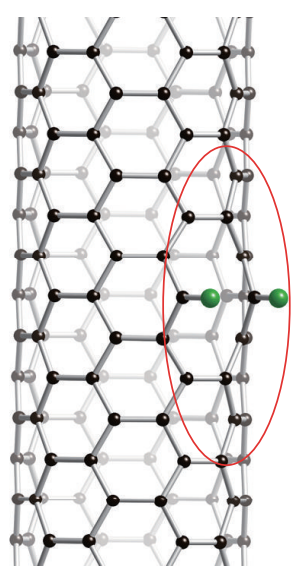

(a)
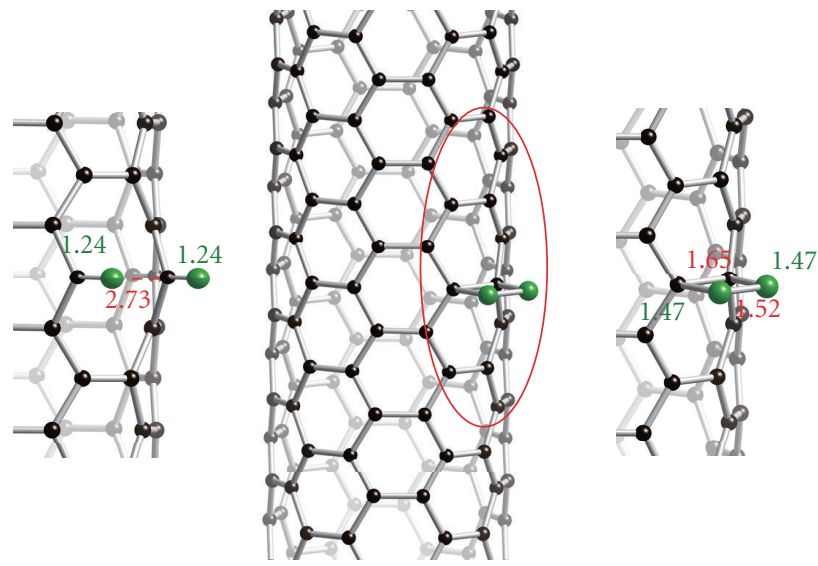

(b)

Figure 3: (a) Optimized structure for the $(5,5)$ nanotube attached by one diketone group, where the CC bond at the binding site opens. (b) The other type of the nanotube attached by one oxygen pair, where the OO bond as well as the CC bond at the binding site retain.

(In the PBC calculations (Figure 2), we relaxed cell parameter in $\mathrm{c}$ vector as well as position of included atoms. However two cell parameters in $\mathrm{a}$ and $\mathrm{b}$ vectors were fixed, and eventually intertube separations are fixed). The intertube separations were large enough to avoid significant intertube interactions [42-44]. The cell size is also large enough to avoid interactions between the guests located on the neighboring unit cells. We employed a $1 \times 1 \times 5 k$-point mesh for geometry optimization of the nanotube systems. In $\mathrm{PBC}$ calculations of metallic systems with no band gap, we sometimes faced a difficulty in converging their total energy. To avoid the difficulty, the convergence was enhanced in the VASP program using fractional occupancy generated by Gaussian broadening $\left(\sigma_{\mathrm{GB}}=0.03 \mathrm{eV}\right)$ of the oneelectron energy levels. As a result, the energy levels close to the Fermi level have fractional occupations. Based on the PW91 calculations, the CC bond length in graphene was determined to be $1.421 \AA$, which is consistent with the experimental value $[1,2]$ suggesting that the method of our choice is appropriate for the present study.

In the finite-length model calculation performed with the Gaussian 03 program [45], we fully optimized a $(5,5)$ nanotube terminated by $\mathrm{H}$ atoms $\left(\mathrm{C}_{230} \mathrm{H}_{20}\right)$ plus $N_{\text {op }}$ oxygen pairs. The length of the tube model was approximately $27 \AA$, which was large enough to model the corresponding infinitelength tube [46-49]. We used the $6-31 G^{*}$ basis set [50] for the attached oxygen pairs as well as the neighboring $58 \mathrm{C}$ atoms, and the 3-21 G basis set for all of the other atoms [51]. Similar to previous theoretical studies [18-20], single and triplet spin states of the diketone-attached nanotubes were obtained for the finite-length calculations.

\section{Results and Discussion}

3.1. Formation of One Diketone Group on a $(5,5)$ Nanotube. As proposed by Kosynkin et al. [26], the diketone formation would be an indispensable reaction to cleave the tube CC bonds at the binding sites. In this section, we describe how DFT calculations were used to investigate relationships between the diketone formation and the CC bond cleavage. For this purpose, we constructed two types of initial geometries for the addition of two oxygen atoms into the $(5,5)$ nanotube, which can be distinguished by whether the attached C-O bonds are longer or shorter. As a result of PW91 optimization, we obtained two local minima, as shown in Figure 3. Figure 3(a) displays the optimized structure for the formation of one diketone group on the $(5,5)$ nanotube. As shown in Figure 3(a), the optimized CO bond length is $1.24 \AA$, which indicated the presence of two CO double bonds (The optimized geometry was obtained by using the initial geometry of the nanotube attached by one oxygen-atom pair with shorter $\mathrm{C}-\mathrm{O}$ bonds). Then, the $\mathrm{CC}$ bond at the binding site is broken (CC separation, $2.73 \AA$ ). Another type of optimized structure was also obtained, and it is shown in Figure 3(b) (The optimized geometry was obtained by using the initial geometry of the nanotube attached by one oxygenatom pair with longer $\mathrm{C}-\mathrm{O}$ bonds). The optimized structure is $31.3 \mathrm{kcal} / \mathrm{mol}$ more unstable compared to the structure in Figure 3(a). A similar value was obtained in [37]. In the oxygen-pair addition shown in Figure 3(b), two single CO bonds $(1.47 \AA)$ are formed. Then, the OO bond $(1.52 \AA)$ stretches, but it does not completely break. The optimized structure retains the tube CC bond at the binding site.

A comparison between the two optimized structures elucidated the roles of the oxygen-pair addition in the CC bond cleavage. To understand the different chemical bonds between the two $\mathrm{C}_{2} \mathrm{O}_{2}$ networks, the valence concept was utilized. Conceptually, the two $\mathrm{C}_{2} \mathrm{O}_{2}$ networks are connected by an electron transfer, which is indicated by the arrows in Figure 4. The green arrow in Figure 4 indicates a singleelectron transfer from the $\mathrm{OO}$ bond to the $\mathrm{CO}$ bond, while a blue arrow indicates a single-electron transfer from the attached CC bond to the CO bond. As a result of the electron transfer, the CC and OO bonds are cleaved, and two double CO bonds are formed (Figure 4). This process can be rationalized with the Mulliken charge populations, and the calculated values are also presented in Figure 4. Thus, 

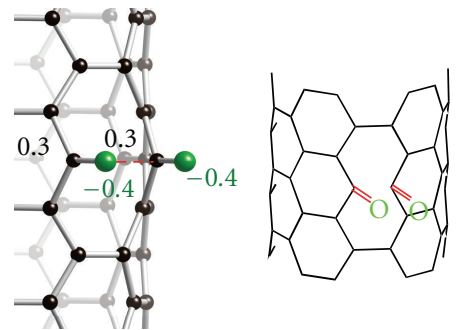

OO bond cleavage CC bond cleavage

Dione formation
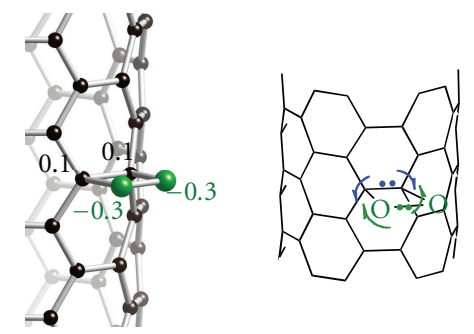

Figure 4: A view of oxygen pair-attached tubes from valence concept, and Muliken charge population.
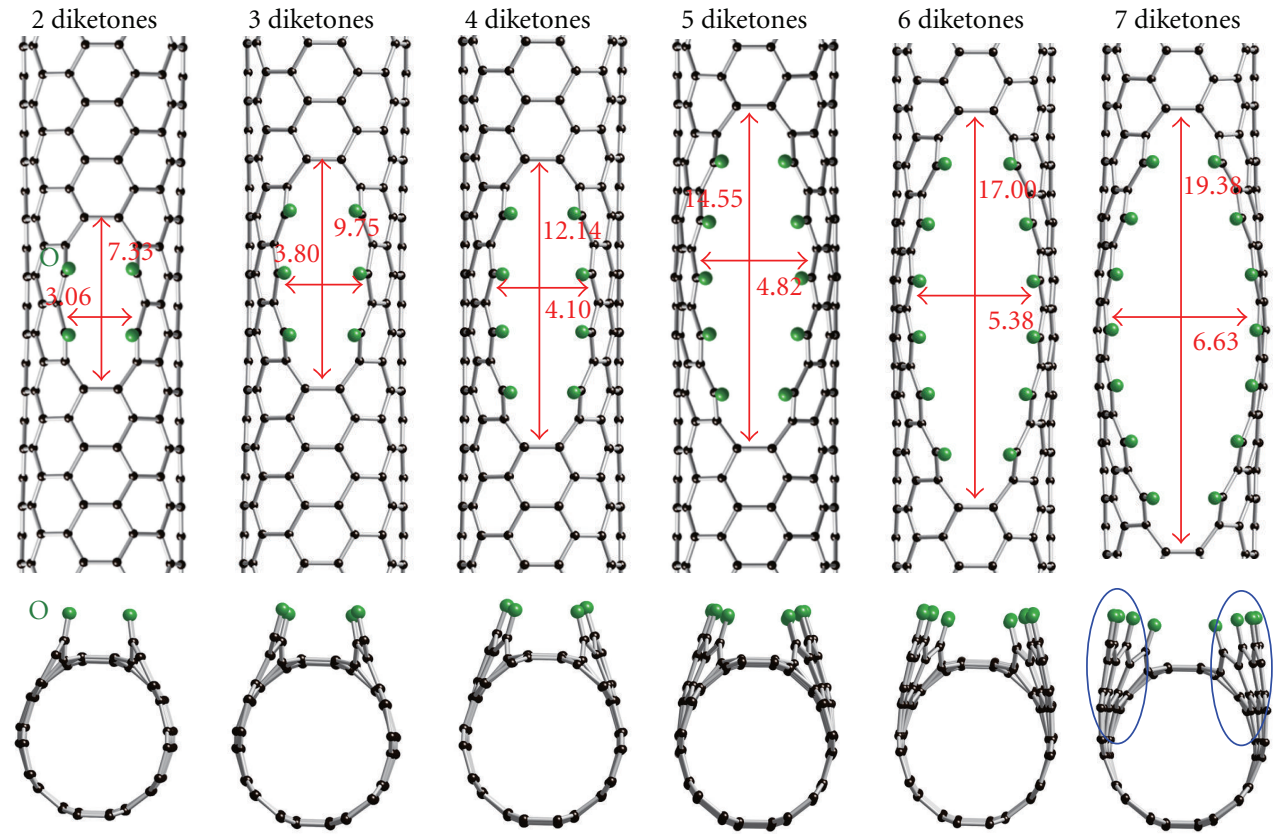

Figure 5: Overview of a series of optimized structures for diketone-attached nanotubes. The number of diketone groups $\left(N_{\mathrm{op}}\right)$ ranges from 2 to 7 .

the DFT results support the experimental prediction posited in [26].

\subsection{A Series of Diketone-Attached Infinite-Length $(5,5)$} Nanotube. In this section, we discuss a series of diketoneattached infinite-length $(5,5)$ nanotube where the number of attached diketone groups $\left(N_{\mathrm{op}}\right)$ ranges from 2 to 7 . Their local minima were obtained from spin-polarized and spinunpolarized DFT calculations. Table 1 shows the relative energy between the spin-polarized and spin-unpolarized solutions of each diketone-attached nanotube $\left(\Delta E_{\text {state }}=\right.$ $\left.E_{\text {polarized }}-E_{\text {unpolarized }}\right)$. The negative $\Delta E_{\text {state }}$ values in Table 1 suggest that a spin-polarized solution is energetically preferable relative to a spin-unpolarized solution. In particular, 3and 4-diketone-attached nanotubes have substantial $\Delta E_{\text {state }}$ values ( -4.2 and $-6.2 \mathrm{kcal} / \mathrm{mol}$, resp.).

Figure 5 provides an overview of the structures for the

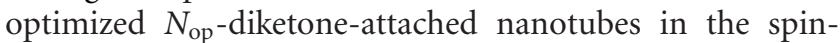
polarized ground state. For comparison, the optimized structures for the corresponding $\mathrm{H}$-attached nanotubes are also displayed in Figure 6. In Figure 5, the attached oxygen (green) atoms are aligned along the tube axis irrespective
TABLE 1: Relative energy between spin-polarized and spin-unpolarized solutions of $N_{\text {op }}$ diketone-attached nanotubes.

\begin{tabular}{|c|c|c|c|c|c|c|}
\hline \multirow{3}{*}{$\Delta E_{\text {state }}{ }^{\mathrm{b}}$} & \multicolumn{6}{|c|}{$N_{\text {op }}{ }^{\mathrm{a}}$} \\
\hline & 2 & 3 & 4 & 5 & 6 & 7 \\
\hline & 0.0 & -4.2 & -6.2 & -1.8 & -3.0 & -2.7 \\
\hline \multicolumn{7}{|c|}{ 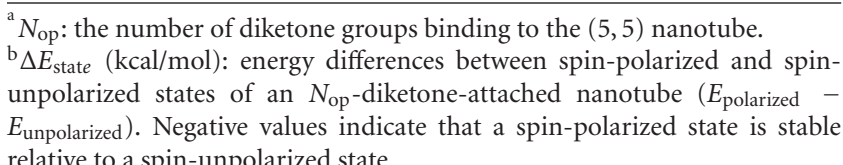 } \\
\hline
\end{tabular}

of the number of attached oxygen atoms. It is important to note that the $\mathrm{OO}$ separations along the tube axis are nearly constant $(\sim 2.5 \AA)$ for larger $N_{\text {op }}$ values. Such geometrical features cannot be found by Rangel et al. [36]. As in the case of the 1-diketone-attached nanotube, the $\mathrm{CC}$ bonds break as the multiple diketone groups are formed on the nanotube. With an increase in $N_{\text {op }}$, larger CC separations are found. Accordingly, a larger hole is generated in the $N_{\text {op }}$-diketone-attached nanotube with a larger $N_{\text {op }}$ value, as shown in Figure 5. For 


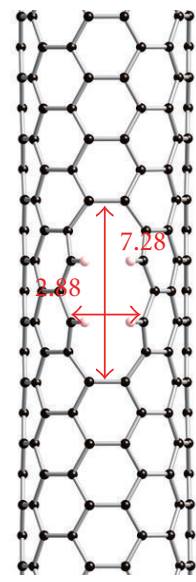

$4 \mathrm{H}$ attached

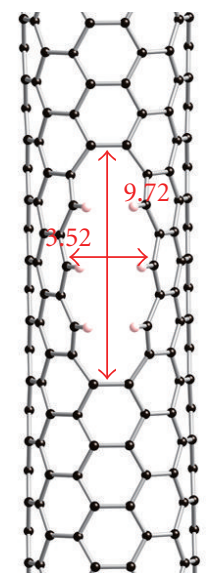

$6 \mathrm{H}$ attached
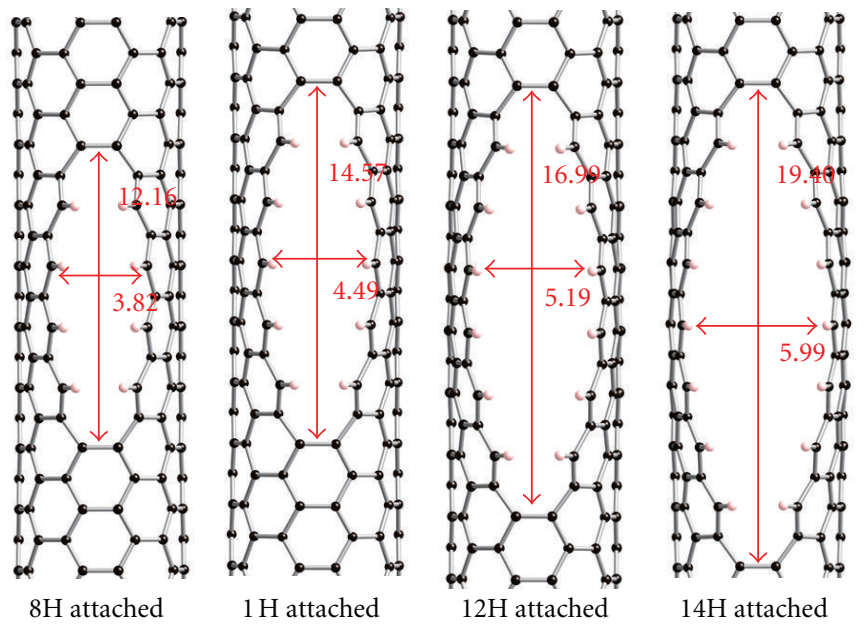

FiguRE 6: Overview of a series of optimized structures for hydrogen-attached nanotubes. The number of attached $\mathrm{H}$ atoms ranges from 4 to 14.
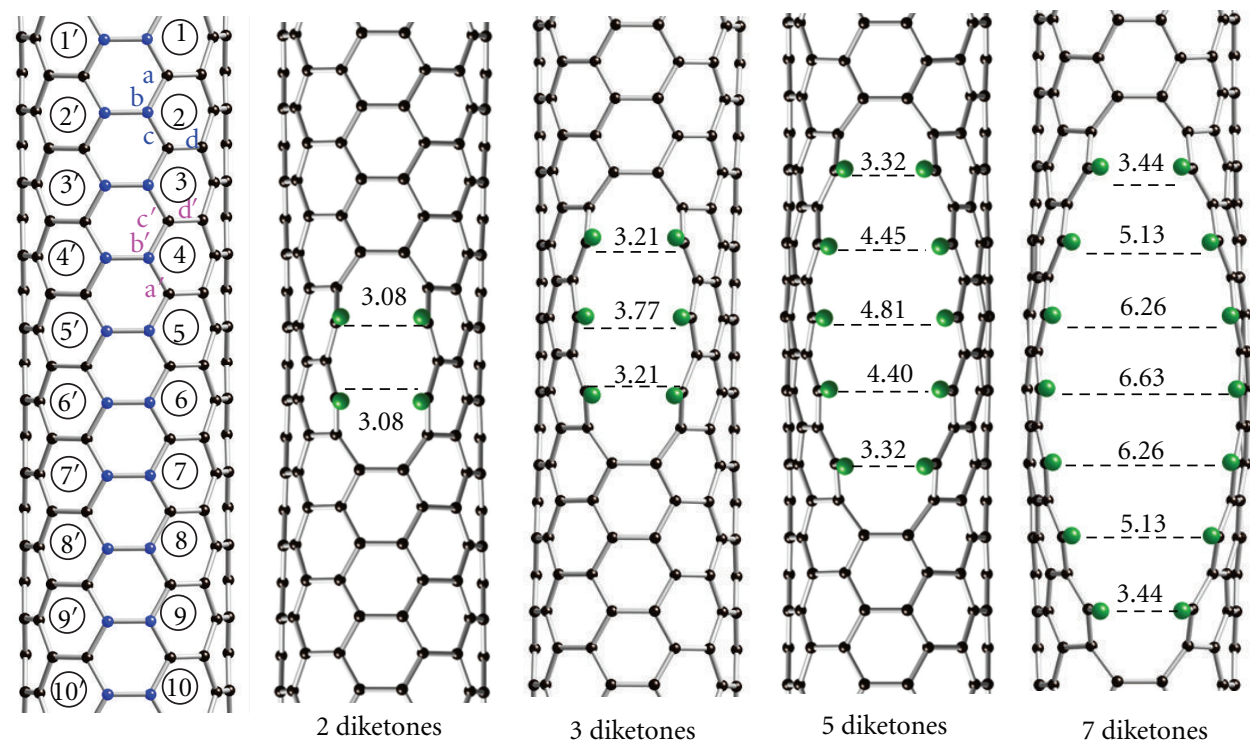

Figure 7: Detailed optimized structures for 2-, 3-, 5-, and 7-diketone-attached nanotubes. We labeled six-membered rings that will be attached by oxygen pairs. Values in $\AA$ indicate the optimized CC separations, created by diketone formation. Dihedral angles of the a, b, c, and $\mathrm{d}$ carbon atoms are defined. Note that the carbon atoms labeled by "b" are the binding site for ketone formation.

example, the 7-diketone-attached nanotube has a hole of $19.4 \times 6.6 \AA$. Interestingly, the hole width $(6.6 \AA)$ is comparable to the diameter of the pristine $(5,5)$ nanotube $(6.9 \AA)$. In terms of the hole formation, similarities were found in the $\mathrm{H}$-attached nanotubes in Figure 6. However, the $\mathrm{H}$-attached nanotube has a slightly smaller hole than the corresponding diketone-attached nanotube case. The different hole sizes can be reproduced in the finite-length calculations. According to Mulliken population analyses in the finite-length calculations, the attached oxygen atoms have substantial negative charges $(-0.38 \mathrm{e} \sim-0.29 \mathrm{e})$ (nanotubes attached by smaller number of oxygen pairs have more significant charges with negative signs). Between the paired oxygen atoms attached to the nanotubes, repulsive forces exert, forming the larger holes in the diketone-attached nanotubes than those in the corresponding $\mathrm{H}$-atom-attached nanotubes where such negative charges do not appear on the attached $\mathrm{H}$-atoms.

Because of the large-hole formation, the 7-diketoneattached nanotube cannot retain a tubular structure. However, some of the six-membered rings seem to be planarized and are shown as circles in Figure 5. To determine how the hole formation results in planarizing of the six-membered rings, we investigate in detail the structural features of the 2-, 3-, 5-, and 7-diketone-attached nanotubes, as shown in Figure 7 . The six-membered rings attached by a ketone group are labeled to identify the binding sites. For example, the binding sites in the 7-diketone-attached nanotube have blue carbon atoms in the six-membered rings with labels ranging from " 3 " to "9." The dependence of the CC bond cleavage on 


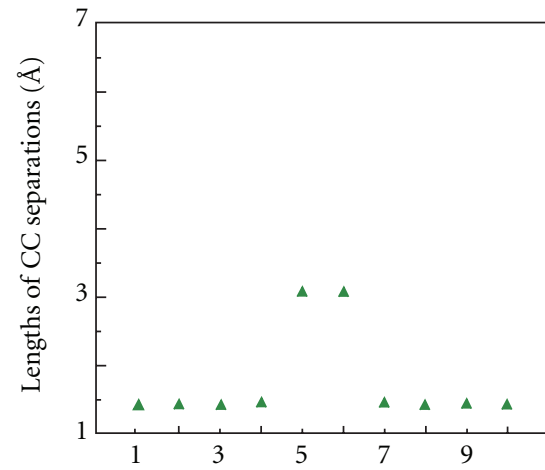

Label number of six-membered rings

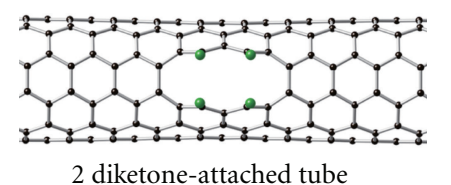

2 diketone-attached tube

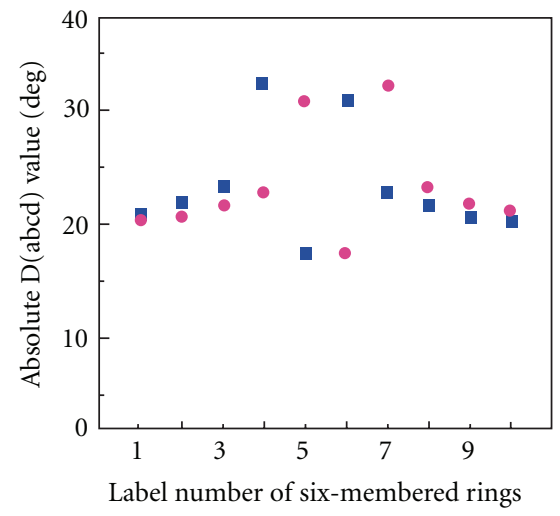

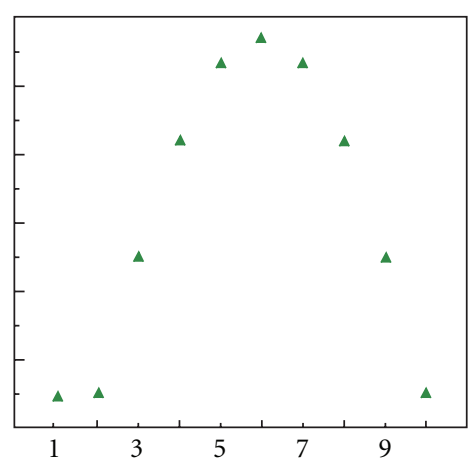

Label number of six-membered rings

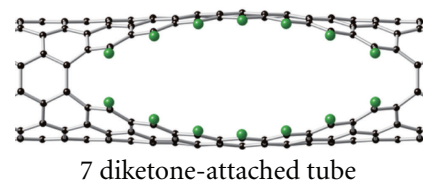

3 diketone-attached tube

(a)
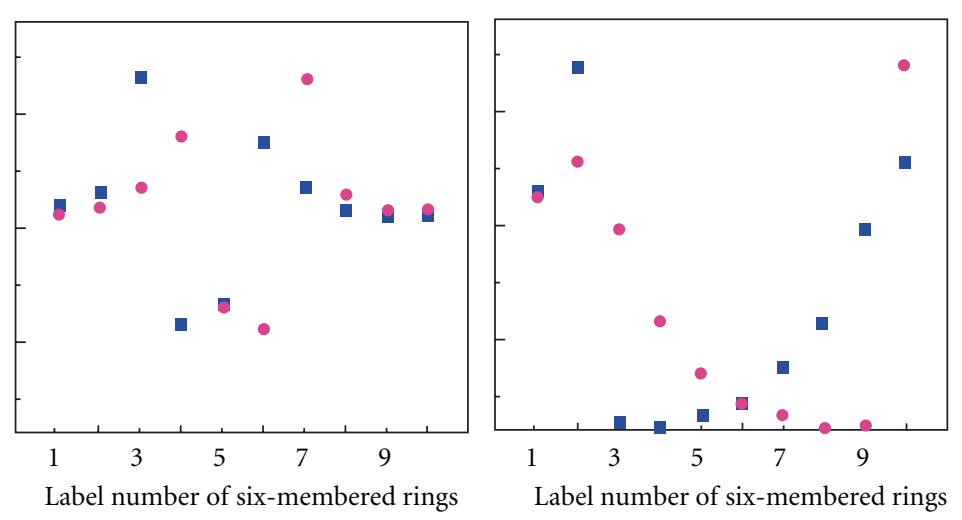

(b)

FIGURE 8: (a) Variations of CC bonds separated by diketone formation, depending on the binding sites. (b) Site dependences of the dihedral angles (D) defined by a, b, c, and d in Figure 7. The absolute D values (in degree) are plotted. The number in the abscissa axis of (a) and (b) corresponds to the label number of six-membered rings in Figure 7.

the location of the attached six-membered rings is displayed in Figures 7 and 8(a). In Figures 7 and 8(a), the two carbon atoms are significantly separated in the nanotubes with larger numbers of attached diketone groups. In particular, the longest CC separation was found in the middle of the hole in the 7-diketone-attached nanotube and is labeled "6" in Figure 7. On the other hand, smaller CC separations were found in the nanotubes attached by a smaller number of diketone groups $\left(N_{\mathrm{op}}=2\right.$ or 3$)$.

Planarization of the six-membered rings surrounding the hole is quantified in Figure 8(b) using the dihedral angles (D) of the four $\mathrm{C}$ atoms $\left(\mathrm{a}, \mathrm{b}, \mathrm{c}\right.$, and $\mathrm{d}\left(\mathrm{a}^{\prime}, \mathrm{b}^{\prime}, \mathrm{c}^{\prime}\right.$, and $\left.\mathrm{d}^{\prime}\right)$ in Figure 7) neighboring the attached site. It is important to note that the pristine $(5,5)$ tube and the planer graphene have absolute D values of 19.8 (Dihedral angles of a, b, c, and $\mathrm{d}$ atoms have positive $\mathrm{D}$ values, whereas those of $\mathrm{a}^{\prime}, \mathrm{b}^{\prime}$, $\mathrm{c}^{\prime}$, and $\mathrm{d}^{\prime \prime}$ have negative values.) and 0 , respectively, as shown in Figure 9. Thus, the dihedral angles are useful parameters for determining if a six-membered ring is planar in the diketone-attached tube. In fact, their $\mathrm{D}$ values would approach 19.8 degrees, when the diketone-attached nanotube exhibits a tubular structure. In contrast, the planarized six-membered rings appear in the diketone-attached nanotube with $\mathrm{D}$ values close to 0 .

As shown in Figure 8(b), we observe an increase in the $\mathrm{D}$ values near the binding sites of the 2-diketone-attached nanotube. In addition to the $\mathrm{D}$ values larger than 19.8 degrees, two six-membered rings have a smaller $\mathrm{D}$ value. Much smaller D values are observed for the 3-diketone-attached nanotube where two diketone-attached rings, labeled 4 and 6, have D values of approximately 10 degrees. Decreasing D values indicate that the curvature of the ring is substantially reduced. When larger numbers of diketone groups are attached on a tube, nearly planar six-membered rings are present. For example, some six-membered rings have $\mathrm{D}$ values ranging from 0 to 10 degrees in the 7 -diketoneattached nanotube. More importantly, Figures 8(a) and 8(b) 


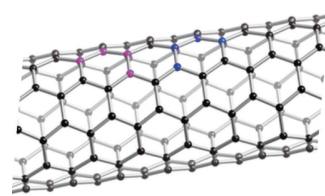

$D($ abcd $)=19.8$

$(5,5)$ tube

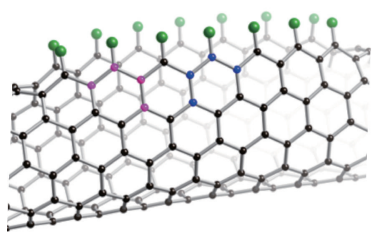

Diketone-attached tube

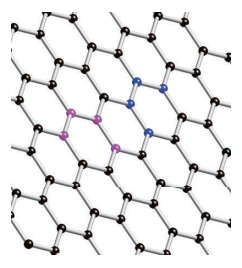

$D($ abcd $)=0$

Graphene

FIGURE 9: Variation of dihedral angle $(\mathrm{D}(\mathrm{abcd}))$ depending on carbon allotropes.
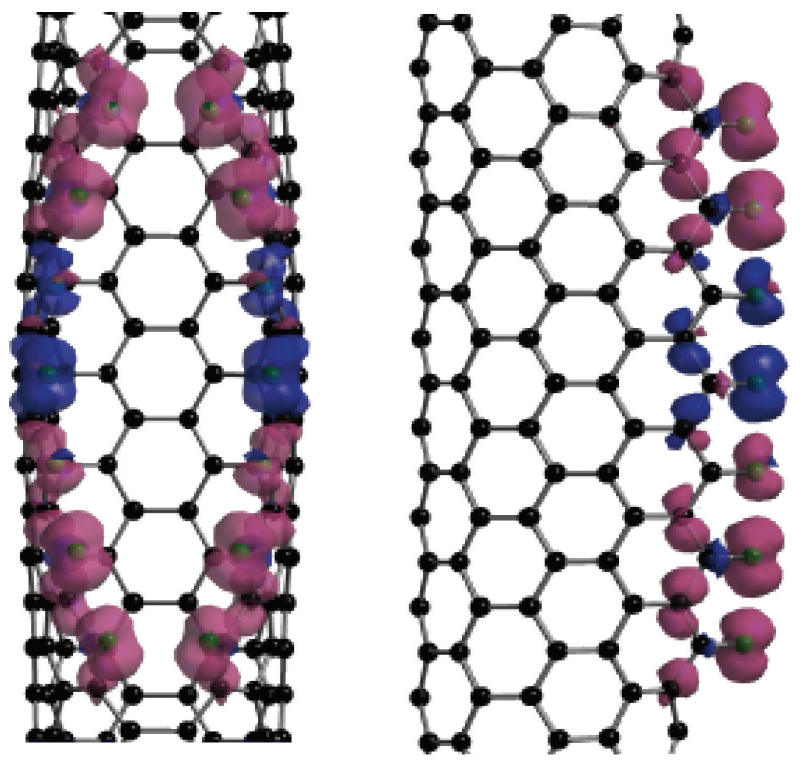

FIGURE 10: Isosurface spin densities of the optimized 7-diketone-attached nanotube in the triplet state. Pink; $\alpha$ spin; blue; $\beta$ spin.

exhibit a clear correlation between the planarity of a sixmembered ring and the dissociation of a CC bond via diketone formation. When carbon atoms are more than $5 \AA$ apart, their $\mathrm{D}$ values are less than 10 degrees. Because the planarized six-membered rings have zigzag edges, the edge structures perturb the electronic properties of the armchair nanotube.

\subsection{Electronic Properties of a Finite-Length Nanotube Attached} by Diketone Groups. In this section, we discuss the electronic properties of the 7-diketone-attached nanotube using a finite-length model. In the finite-length calculations, we obtained results similar to the infinite-length cases in terms of their geometrical features. Furthermore, the 7-diketoneattached nanotube in the triplet state is $5 \mathrm{kcal} / \mathrm{mol}$ more stable than that the singlet state. Because of the stability of the triplet state, the 7-diketone-attached nanotube has radical character. According to the DFT calculations, the unpaired electrons are primarily localized on the diketone $\mathrm{O}$ atoms, as shown in Figure 10, where isosurface $\alpha$ - and $\beta$-spin densities are given by pink and blue, respectively. To understand the unique radical character, we investigated representative $\alpha$-spin orbitals in the frontier orbital region of the 7-diketone-attached nanotube in Figure 11. The HOMO-2 and HOMO- 4 are only 0.19 and $0.34 \mathrm{eV}$ below the HOMO. These orbitals have amplitudes localized on diketone-O atoms, which is not the case for pristine and $\mathrm{H}$-attached [32-35] nanotubes. Similar orbital features are observed in the magnetic orbitals of ketone-attached nanoribbons [20]. Therefore, the multiple diketone attachments yield an armchair nanotube orbital properties similar to those in the corresponding zigzag nanoribbon.

The patterns of the localized orbitals are reminiscent of an iso-distant one-dimensional chain, whose molecular orbital can be given by the following equation:

$$
\psi_{j}=\sqrt{\frac{2}{n+1}} \sum_{q=1}^{n} \sin \left(\frac{\pi j q}{n+1}\right) \chi_{q}
$$

where $n$ is the number of atoms contained in the chain, $\chi_{q}$ is an atomic orbital on the $q$ th atom, and $\Psi_{j}$ is the $j$ th molecular orbital. As mentioned above, the diketone-attached nanotube has two chains with seven oxygen atoms occurring at nearly regular intervals. Thus, their orbital features can be roughly understood from (1). In these systems, $\chi_{q}$ corresponds to oxygen $\mathrm{p}(\mathrm{p}(\mathrm{O}))$ orbitals parallel to or perpendicular to a plane containing a newly formed zigzag edge, which is depicted schematically in Figure 12. For example, the HOMO-2 consists of perpendicular $\mathrm{p}(\mathrm{O})$ orbitals, whereas the HOMO-4 consists of parallel $\mathrm{p}(\mathrm{O})$ orbitals. It 


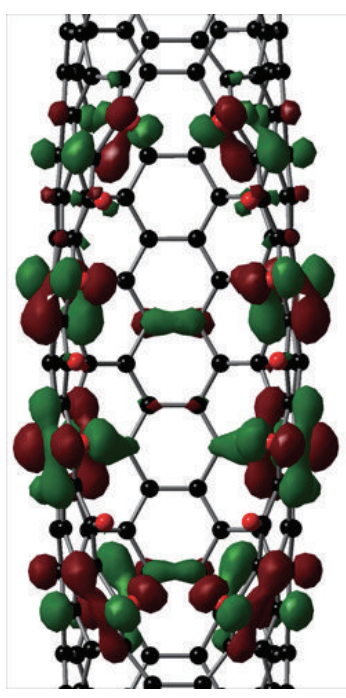

(a) HOMO-2 $(-0.19 \mathrm{eV})$

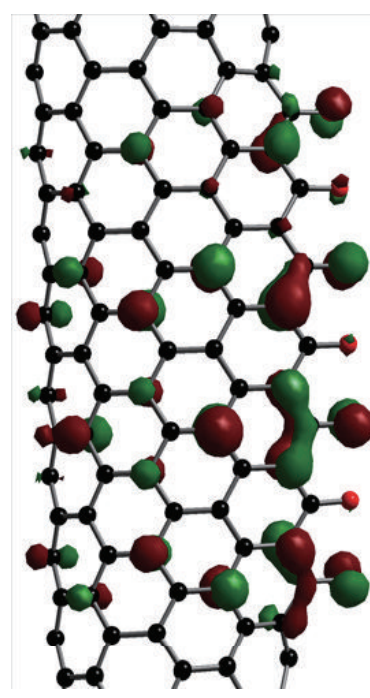

$$
\mathrm{eV})
$$

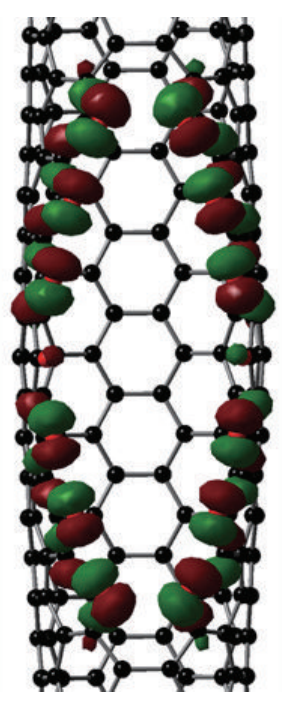

(b) HOMO-4 $(-0.34 \mathrm{eV})$

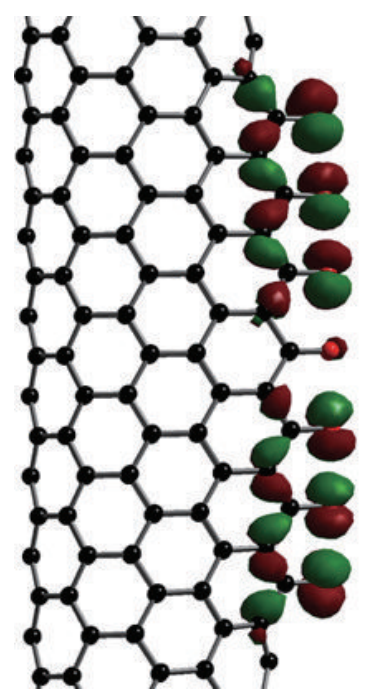

energy value in parentheses FIGURE 11: Representative $\alpha$-spin orbitals of the 7-diketone-attached nanotube in
indicates the energy difference between the $\alpha$-spin HOMO and a certain orbital.

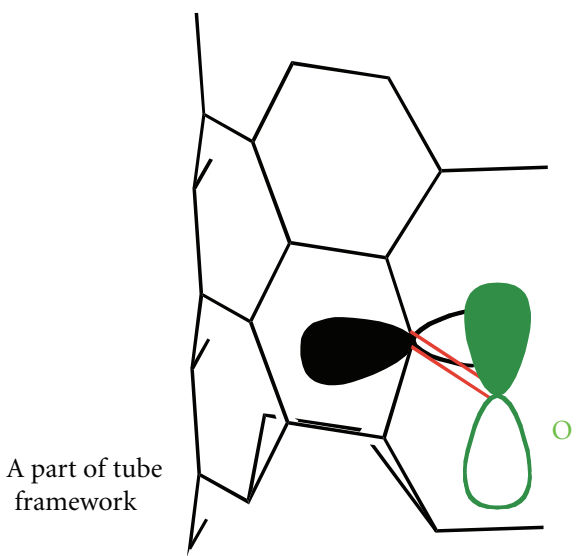

Mismatching orbitals

(a)

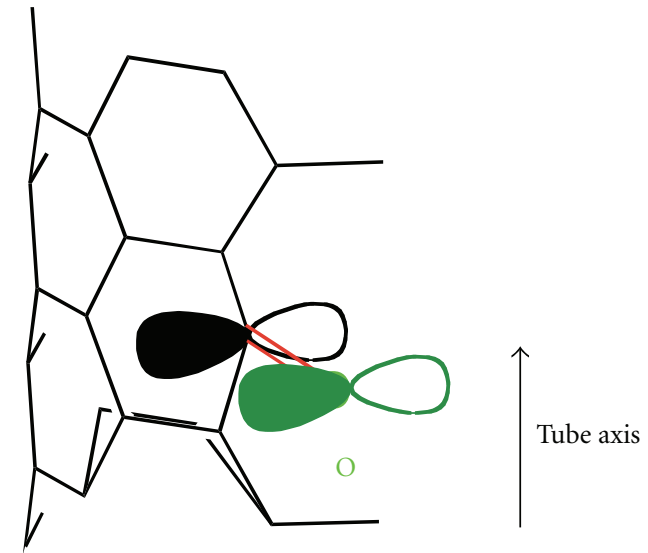

Matching orbitals

(b)

Figure 12: Couplings between an oxygen p orbital and a tube p orbital.

is interesting to note that the localized orbitals have nodes resulting from the sine terms in (1). For example, the HOMO-2 has three nodes along an oxygen-chain, and the HOMO-4 has one node.

Based on the $\mathrm{p}(\mathrm{O})$ orbital orientation in the localized orbitals, the two types of orbitals have different distributions of $\pi$ orbitals in the tube moiety. In fact, the HOMO-2 has carbon $\mathrm{p}$ orbitals slightly distributed into the tube. On the other hand, the HOMO-4 does not have p orbitals on the tube $\mathrm{C}$ atoms, except for carbon atoms that are next-nearest neighbor to a diketone oxygen atom. The different behaviors can be understood from Figure 12 [52]. When parallel $\mathrm{p}(\mathrm{O})$ orbitals are perpendicular to the tube $\pi$ orbitals, they cannot mix with the tube $\pi$ orbitals. As a result, localized orbitals containing parallel $\mathrm{p}(\mathrm{O})$ orbitals do not exhibit amplitudes on the tube moiety. In contrast, perpendicular $\mathrm{p}(\mathrm{O})$ orbitals can interact with the tube $\pi$ orbitals. Because of the interactions, some amplitudes appear in the tube moiety in the frontier orbital containing perpendicular $\mathrm{p}(\mathrm{O})$ orbitals. Consequently, the planarization of the six-membered rings with multiple diketone attachments plays a significant role in determining the orbital features in the frontier orbital region.

Detailed analyses of the frontier orbitals revealed that HOMO- 4 is occupied by an $\alpha$ electron, but it is not occupied by a $\beta$ electron. Therefore, the $\alpha$ HOMO- 4 is one of the key orbitals that determines the spin density distribution in the 7-diketone-attached nanotube. However, there are many frontier orbitals with amplitudes that exist only on the diketone groups. In fact, nine $\alpha$-orbitals lie less than $0.5 \mathrm{eV}$ below the HOMO. These orbitals can be distinguished by the number of nodes as well as the orientation of the $\mathrm{p}(\mathrm{O})$ orbitals involved (e.g., perpendicular or parallel orientation). 
Because many magnetic orbitals exist in the frontier orbital region, we cannot identify all of the orbitals that determine the spin density distributions in the diketone-attached nanotube. However, localized orbitals resulting from p oxygen atoms, such as the HOMO-4, can contribute to the localized radical character.

\section{Conclusions}

Density functional theory (DFT) PW91 calculations were employed to investigate the properties of a $(5,5)$ nanotube attached to some number of diketone groups. According to the DFT calculations, the formation of two double CO bonds on the nanotube cleaves one CC bond at the attachment site. For the high degree of diketone formation on the armchair nanotube, the CC bonds at the binding sites lengthen significantly, and, eventually, a large hole is formed. Then, the sixmembered rings surrounding the large hole are substantially planarized. Because the planarized six-membered rings have zigzag edges attached to diketone groups, the functionalized armchair nanotubes exhibit electronic properties similar to those in nanographene ribbons with zigzag edges. In fact, orbitals with amplitudes primarily localized at diketone groups appear in the frontier orbital region. Based on the orbital features, diketone-attached nanotubes have a spinpolarized ground state, with the unpaired electrons localized on the oxygen atoms

\section{Acknowledgments}

The project is partially supported by a Grant-in-Aid for Young Scientists (B) from the Japan Society for the Promotion of Science (JSPS) (T. Yumura at the Kyoto Institute of Technology) (no. 22710088) and a Grant-in-Aid for Scientific Research (C) from JSPS (T. Yumura at the Kyoto Institute of Technology) (no. 23560934).

\section{References}

[1] M. S. Dresselhaud, G. Dresselhaus, and P. C. Eklund, Science of Fullerenes and Carbon Nanotubes, Academic Press, San Diego, Calif, USA, 1996.

[2] R. Saito, G. Dresselhaus, and M. S. Dresselhaus, Physical Properties of Carbon Nanotubes, Imperial College Press, London, UK, 1998 .

[3] S. Iijima, "Helical microtubules of graphitic carbon," Nature, vol. 354 , no. 6348 , pp. 56-58, 1991.

[4] S. Iijima and T. Ichihashi, "Single-shell carbon nanotubes of 1nm diameter," Nature, vol. 363, no. 6430, pp. 603-605, 1993.

[5] K. S. Novoselov, A. K. Geim, S. V. Morozov et al., "Electric field in atomically thin carbon films," Science, vol. 306, no. 5696, pp. 666-669, 2004.

[6] J. W. Mintmire, B. I. Dunlap, and C. T. White, "Are fullerene tubules metallic?” Physical Review Letters, vol. 68, no. 5, pp. 631-634, 1992.

[7] R. Saito, M. Fujita, G. Dresselhaus, and M. S. Dresselhaus, "Electronic structure of chiral graphene tubules," Applied Physics Letters, vol. 60, no. 18, pp. 2204-2206, 1992.

[8] K. Tanaka, K. Okahara, M. Okada, and T. Yamabe, "Electronic properties of bucky-tube model," Chemical Physics Letters, vol. 191, no. 5, pp. 469-472, 1992.
[9] N. Hamada, S. I. Sawada, and A. Oshiyama, "New one-dimensional conductors: graphitic microtubules," Physical Review Letters, vol. 68, no. 10, pp. 1579-1581, 1992.

[10] M.-H. Whangbo, R. Hoffmann, and R. B. Woodward, "Conjugated one and two dimensional polymers," Proceedings of the Royal Society of London A, vol. 366, no. 1724, pp. 23-46, 1979.

[11] T. Yamabe, K. Tanaka, K. Ohzeki, and S. Yata, "Electronic structure of polyacenacene. A one-dimensional graphite," Solid State Communications, vol. 44, no. 6, pp. 823-825, 1982.

[12] M. Kertesz and R. Hoffmann, "Higher order Peierls distortion of one-dimensional carbon skeletons," Solid State Communications, vol. 47, no. 2, pp. 97-102, 1983.

[13] K. Yoshizawa, K. Yahara, K. Tanaka, and T. Yamabe, "Bandgap oscillation in polyphenanthrenes," Journal of Physical Chemistry B, vol. 102, no. 3, pp. 498-506, 1998.

[14] K. Nakada, M. Fujita, G. Dresselhaus, and M. S. Dresselhaus, "Edge state in graphene ribbons: nanometer size effect and edge shape dependence," Physical Review B, vol. 54, no. 24, pp. 17954-17961, 1996.

[15] K. Kusakabe and M. Maruyama, "Magnetic nanographite," Physical Review B, vol. 67, no. 9, Article ID 092406, 2003.

[16] M. Fujita, K. Wakabayashi, K. Nakada, and K. Kusakabe, "Peculiar localized state at zigzag graphite edge," Journal of the Physical Society of Japan, vol. 65, no. 7, pp. 1920-1923, 1996.

[17] K. Wakabayashi, M. Sigrist, and M. Fujita, "Spin wave mode of edge-localized magnetic states in nanographite zigzag ribbons," Journal of the Physical Society of Japan, vol. 67, no. 6, pp. 2089-2093, 1998.

[18] O. Hod, V. Barone, J. E. Peralta, and G. E. Scuseria, "Enhanced half-metallicity in edge-oxidized zigzag graphene nanoribbons," Nano Letters, vol. 7, no. 8, pp. 2295-2299, 2007.

[19] H. Zheng and W. Duley, "First-principles study of edge chemical modifications in graphene nanodots," Physical Review B, vol. 78, no. 4, Article ID 045421, 2008.

[20] G. Lee and K. Cho, "Electronic structures of zigzag graphene nanoribbons with edge hydrogenation and oxidation," Physical Review B, vol. 79, no. 16, Article ID 165440, 2009.

[21] T. Yumura, K. Kimura, H. Kobayashi, R. Tanaka, N. Okumura, and T. Yamabe, "The use of nanometer-sized hydrographene species for support material for fuel cell electrode catalysts: a theoretical proposal," Physical Chemistry Chemical Physics, vol. 11, no. 37, pp. 8275-8284, 2009.

[22] X. Y. Hu, H. W. Tian, W. T. Zheng et al., "Metallic-semiconducting phase transition of the edge-oxygenated armchair graphene nanoribbons," Chemical Physics Letters, vol. 501, no. 1-3, pp. 64-67, 2010.

[23] T. Yumura, H. Kobayashi, and T. Yamabe, "Roles of radical characters of pristine and nitrogen-substituted hydrographene in dioxygen bindings," Journal of Chemical Physics, vol. 133, no. 17, Article ID 174703, 2010.

[24] A. L. Higginbotham, D. V. Kosynkin, A. Sinitskii, Z. Sun, and J. M. Tour, "Lower-defect graphene oxide nanoribbons from multiwalled carbon nanotubes," ACS Nano, vol. 4, no. 4, pp. 2059-2069, 2010.

[25] D. V. Kosynkin, W. Lu, A. Sinitskii, G. Pera, Z. Sun, and J. $\mathrm{M}$. Tour, "Highly conductive graphene nanoribbons by longitudinal splitting of carbon nanotubes using potassium vapor," ACS Nano, vol. 5, no. 2, pp. 968-974, 2011.

[26] D. V. Kosynkin, A. L. Higginbotham, A. Sinitskii et al., "Longitudinal unzipping of carbon nanotubes to form graphene nanoribbons," Nature, vol. 458, no. 7240, pp. 872-876, 2009.

[27] A. Sinitskii, A. A. Fursina, D. V. Kosynkin, A. L. Higginbotham, D. Natelson, and J. M. Tour, "Electronic transport in monolayer graphene nanoribbons produced by chemical unzipping 
of carbon nanotubes," Applied Physics Letters, vol. 95, no. 25, Article ID 253108, 2009.

[28] L. Jiao, L. Zhang, X. Wang, G. Diankov, and H. Dai, "Narrow graphene nanoribbons from carbon nanotubes," Nature, vol. 458, no. 7240, pp. 877-880, 2009.

[29] A. L. Elas, A. R. Botello-Mndez, D. Menese-Rodrguez et al., "Longitudinal cutting of pure and doped carbon nanotubes to form graphitic nanoribbons using metal clusters as nanoscalpels," Nano Letters, vol. 10, no. 2, pp. 366-372, 2010.

[30] A. G. Cano-Mrquez, F. J. Rodrguez-Macas, J. CamposDelgado et al., "Ex-MWNTs: graphene sheets and ribbons produced by lithium intercalation and exfoliation of carbon nanotubes," Nano Letters, vol. 9, no. 4, pp. 1527-1533, 2009.

[31] X. Wang, "Deformation guided unzipping of nanotubes: a first-principles study," Physics Letters A, vol. 374, no. 48, pp. 4894-4898, 2010.

[32] B. Huang, Y.-W. Son, G. Kim, W. Duan, and J. Ihm, "Electronic and magnetic properties of partially open carbon nanotubes," Journal of the American Chemical Society, vol. 131, no. 49, pp. 17919-17925, 2009.

[33] B. Wang and J. Wang, "First-principles investigation of transport properties through longitudinal unzipped carbon nanotubes," Physical Review B, vol. 81, no. 4, Article ID 045425, 2010.

[34] H. Santos, L. Chico, and L. Brey, "Carbon nanoelectronics: unzipping tubes into graphene ribbons," Physical Review Letters, vol. 103, no. 8, Article ID 086801, 2009.

[35] B. Huang, Z. Li, Y.-W. Son, G. Kim, W. Duan, and J. Ihm, "Multiple localized states and magnetic orderings in partially open zigzag carbon nanotube superlattices: an ab initio study," Journal of Chemical Physics, vol. 133, no. 8, Article ID 084702, 2010.

[36] N. L. Rangel, J. C. Sotelo, and J. M. Seminario, "Mechanism of carbon nanotubes unzipping into graphene ribbons," Journal of Chemical Physics, vol. 131, no. 3, Article ID 031105, 2009.

[37] H. Zhang, M. Zhao, T. He et al., "Orientation-selective unzipping of carbon nanotubes," Physical Chemistry Chemical Physics, vol. 12, no. 41, pp. 13674-13680, 2010.

[38] J. Wang, L. Ma, Q. Yuan, L. Zhu, and F. Ding, "Transitionmetal-catalyzed unzipping of single-walled carbon nanotubes into narrow graphene nanoribbons at low temperature," Angewandte Chemie, vol. 50, no. 35, pp. 8041-8045, 2011.

[39] J. P. Perdew and Y. Wang, "Accurate and simple analytic representation of the electron-gas correlation energy," Physical Review B, vol. 45, no. 23, pp. 13244-13249, 1992.

[40] G. Kresse and J. Furthmüller, "Efficient iterative schemes for ab initio total-energy calculations using a plane-wave basis set," Physical Review B, vol. 54, no. 16, pp. 11169-11186, 1996.

[41] D. Vanderbilt, "Soft self-consistent pseudopotentials in a generalized eigenvalue formalism," Physical Review B, vol. 41, no. 11, pp. 7892-7895, 1990.

[42] T. Yumura, M. Kertesz, and S. Iijima, "Confinement effects on site-preferences for cycloadditions into carbon nanotubes," Chemical Physics Letters, vol. 444, no. 1-3, pp. 155-160, 2007.

[43] T. Yumura and M. Kertesz, "Cooperative behaviors in carbene additions through local modifications of nanotube surfaces," Chemistry of Materials, vol. 19, no. 5, pp. 1028-1034, 2007.

[44] T. Yumura and M. Kertesz, "Roles of conformational restrictions of a bismalonate in the interactions with a carbon nanotube," Journal of Physical Chemistry C, vol. 113, no. 32, pp. 14184-14194, 2009.

[45] M. J. Frisch et al., Gaussian 03, Gaussian, Pittsburgh, Pa, USA, 2003.

[46] T. Yumura, K. Hirahara, S. Bandow, K. Yoshizawa, and S. Iijima, "A theoretical study on the geometrical features of finite-length carbon nanotubes capped with fullerene hemisphere," Chemical Physics Letters, vol. 384, no. 4-6, pp. 320325, 2004.

[47] T. Yumura, S. Bandow, K. Yoshizawa, and S. Iijima, "The role of fullerene hemispheres in determining structural features of finite-length carbon nanotubes," Journal of Physical Chemistry B, vol. 108, no. 31, pp. 11426-11434, 2004.

[48] T. Yumura, D. Nozaki, S. Bandow, K. Yoshizawa, and S. Iijima, "End-cap effects on vibrational structures of finite-length carbon nanotubes," Journal of the American Chemical Society, vol. 127, no. 33, pp. 11769-11776, 2005.

[49] T. Yumura, M. Kertesz, and S. Iijima, "Local modifications of single-wall carbon nanotubes induced by bond formation with encapsulated fullerenes," Journal of Physical Chemistry B, vol. 111, no. 5, pp. 1099-1109, 2007.

[50] W. J. Hehre, K. Ditchfield, and J. A. Pople, "Self-consistent molecular orbital methods. XII. Further extensions of gaussian-type basis sets for use in molecular orbital studies of organic molecules," The Journal of Chemical Physics, vol. 56, no. 5, pp. 2257-2261, 1972.

[51] J. S. Binkley, J. A. Pople, and W. J. Hehre, "Self-consistent molecular orbital methods. 21. Small split-valence basis sets for first-row elements," Journal of the American Chemical Society, vol. 102, no. 3, pp. 939-947, 1980.

[52] T. A. Albright, J. K. Burdett, and M.-H. Whangbo, Orbital Interactions in Chemistry, A Wiley-Interscience, New York, NY, USA, 1984. 

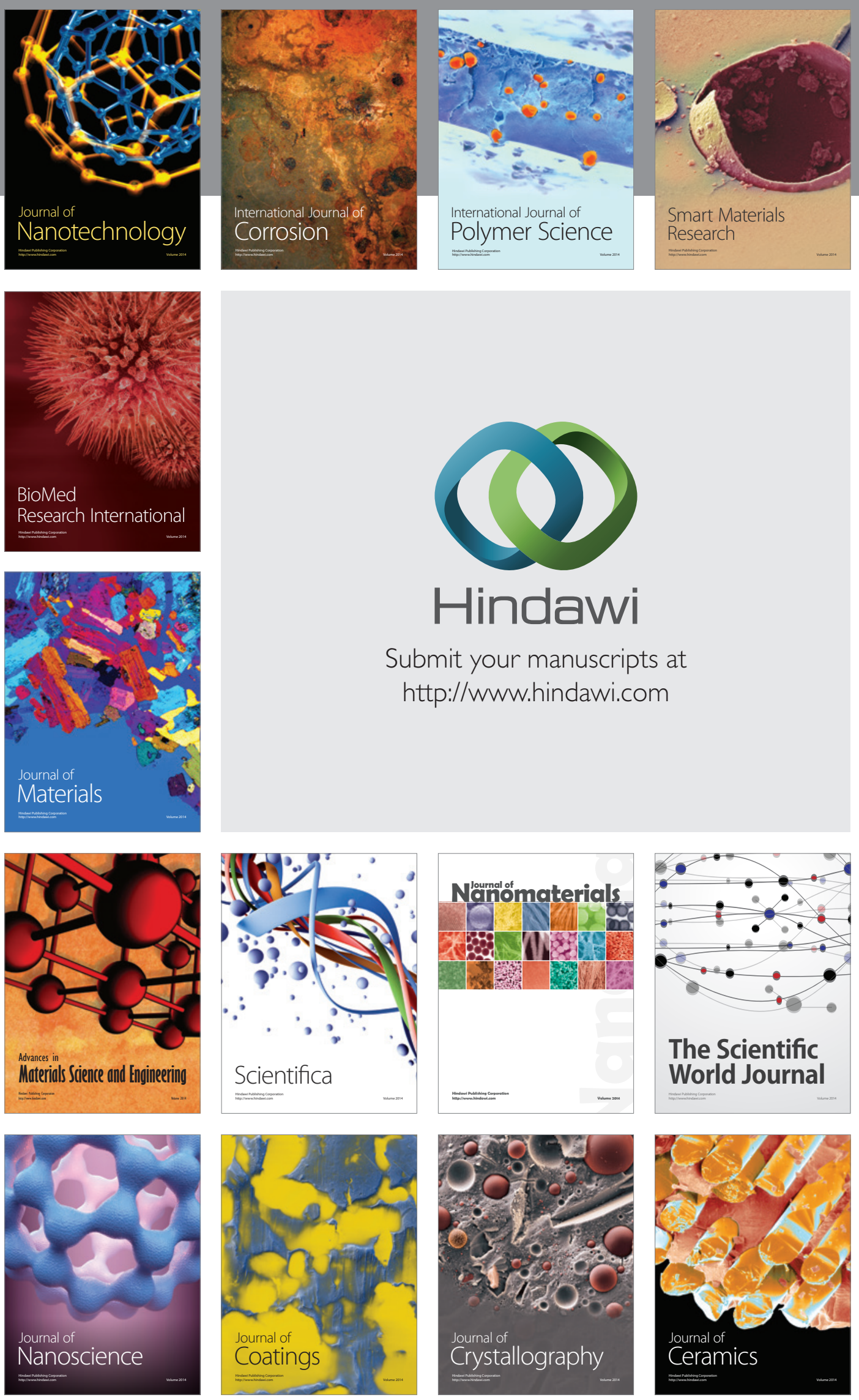

The Scientific World Journal

Submit your manuscripts at

http://www.hindawi.com

\section{World Journal}

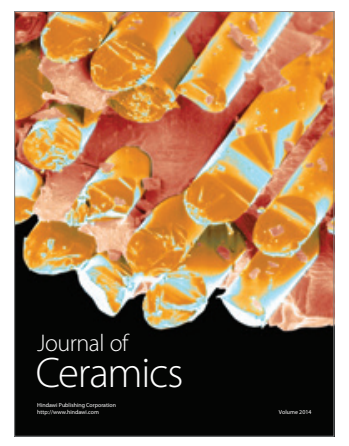

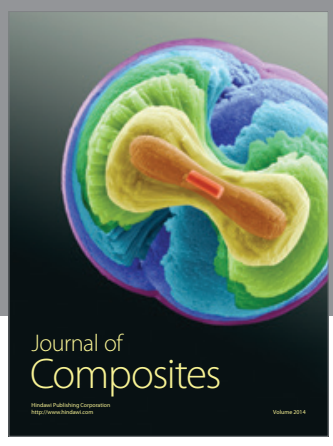
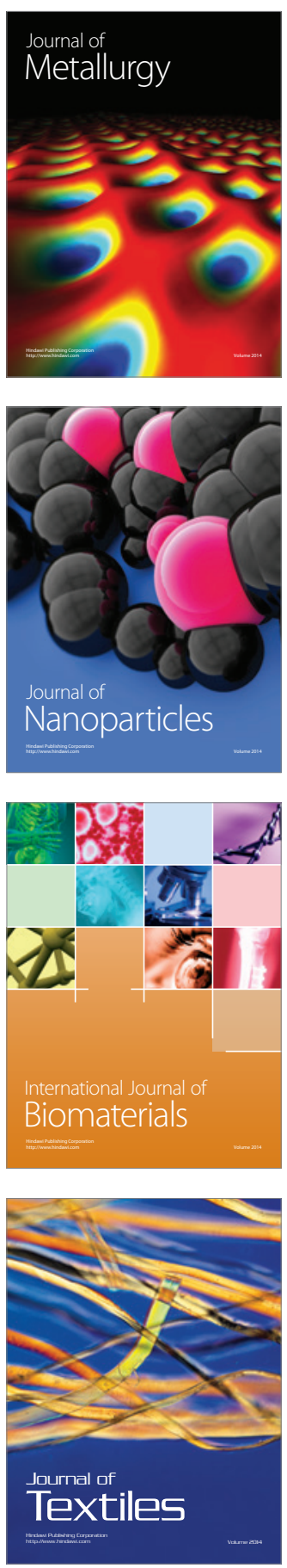